\title{
Effects of different finishing/polishing protocols and systems for monolithic zirconia on surface topography, phase transformation, and biofilm formation
}

\author{
Hang-Nga Mai ${ }^{1}$, Su-Hyung Hong ${ }^{2}$, Sung-Hun Kim ${ }^{3}$, Du-Hyeong Lee ${ }^{1 *}$ \\ 'Department of Prosthodontics, School of Dentistry, ITRD, Kyungpook National University, Daegu, Republic of Korea \\ ${ }^{2}$ Department of Microbiology and Immunology, School of Dentistry, Kyungpook National University, Daegu, Republic of Korea \\ ${ }^{3}$ Department of Prosthodontics, School of Dentistry, Seoul National University, Seoul, Republic of Korea
}

\begin{abstract}
PURPOSE. The purpose of this study was to evaluate the effects of various protocols and systems for finishing and polishing monolithic zirconia on surface topography, phase transformation, and bacterial adhesion. MATERIALS AND METHODS. Three hundred monolithic zirconia specimens were fabricated and then treated with three finishing and polishing systems (Jota [JO], Meisinger [ME], and Edenta [ED]) using four surface treatment protocols: coarse finishing alone (C); coarse finishing and medium polishing (CM); coarse finishing and fine polishing (CF); and coarse finishing, medium polishing, and fine polishing (CMF). Surface roughness, crystal phase transformation, and bacterial adhesion were evaluated using atomic force microscopy, X-ray diffraction, and streptococcal biofilm formation assay, respectively. One-way and two-way analysis of variance with Tukey post hoc tests were used to analyze the results $(\alpha=.05)$. RESULTS. In this study, the surface treatment protocols and systems had significant effects on the resulting roughness. The CMF protocol produced the lowest roughness values, followed by CM and CF. Use of the JO system produced the lowest roughness values and the smallest biofilm mass, while the ME system produced the smallest partial transformation ratio. The ED group exhibited the highest roughness values, biofilm mass, and partial transformation ratio. CONCLUSION. Stepwise surface treatment of monolithic zirconia, combined with careful polishing system selection, is essential to obtaining optimal microstructural and biological surface results. [J Adv Prosthodont 2019;11:81-7]
\end{abstract}

KEYWORDS: Zirconia; Dental finishing; Dental polishing; Roughness; Biofilm

\section{INTRODUCTION}

Monolithic zirconia restorations have been widely used because of their excellent mechanical strength and biocom-

Corresponding author:

Du-Hyeong Lee

Department of Prosthodontics, School of Dentistry, Kyungpook National University, 2175 Dalgubeoldae-ro, Jung-gu, Daegu 41940, Republic of Korea Tel. +82536007676: e-mail, deweylee@knu.ac.kr

Received July 10, 2018 / Last Revision October 31, 2018 / Accepted November 18, 2018

(C) 2019 The Korean Academy of Prosthodontics

This is an Open Access article distributed under the terms of the Creative Commons Attribution Non-Commercial License (http://creativecommons. org/licenses/by-nc/4.0) which permits unrestricted non-commercial use, distribution, and reproduction in any medium, provided the original work is properly cited.

This work was supported by the National Research Foundation of Korea (NRF) grant funded by the Korea government (MSIP) (2017R1C1B2004976). patibility. ${ }^{1-3}$ Clinical adjustment of zirconia restorations is necessary when a restoration exhibits premature occlusal contacts or inadequate contours on delivery. ${ }^{4-6}$ The grinding process during adjustment removes the polished layer and results in rough surfaces, ${ }^{7}$ which may cause clinical complications such as wear of the antagonistic dentition, ${ }^{8}$ accumulation of dental plaque, ${ }^{9}$ and color staining. ${ }^{10}$ Therefore, further surface smoothing is necessary before the restoration is placed in the mouth.

Glazing and polishing are two options for surface smoothing of zirconia restorations. ${ }^{11,12}$ Although glazing is a popular method for restoring the high-gloss surface of the restoration, glazed layers can reportedly become worn within 6 months of the restoration. ${ }^{12}$ The polishing procedure, on the other hand, does not add any layer to the surface of the monolithic zirconia restoration. The polishing alone removes the material by abrasion in which polishing abra- 
sives transmit the mechanical energy to the material, resulting in changing of the surface roughness. ${ }^{6}$ Moreover, this method can produce surface roughness of $0.2 \mu \mathrm{m}$, which less than or equal to that achieved with glazing., ${ }^{7,13}$ Surface roughness $\leq 0.2 \mu \mathrm{m}$ provides minimal plaque accumulation and comfortable tactile sensation.9,14 Therefore, zirconia polishing may be an effective and time-saving alternative to glazing. ${ }^{15,16}$

Surface smoothing can be subdivided into three major stages: coarse finishing, intermediate polishing, and final polishing. ${ }^{17}$ Coarse finishing uses coarse-grit grinding instruments to obtain the desired contours of the restoration by removing excess restorative materials. Intermediate polishing uses medium-grit instruments to level the grooves and scratches created by the coarse-finishing process. Final polishing aims to produce a very smooth, light-reflective, enamel-like surface on the restoration through use of fine-grit instruments. To achieve the desired smoothness, it is recommended that instruments be employed sequentially from coarse to fine with different revolutions per minute (RPM) for each step. ${ }^{18,19}$

Zirconium-dioxide exists in three crystalline phases, depending on the temperature. ${ }^{2}$ At room temperature, zirconia usually exists in a monoclinic phase, which has a lower density than the other phases. By heating zirconia to $1170^{\circ} \mathrm{C}$, the monoclinic phase transforms into the tetragonal phase, which provides better mechanical properties. The tetragonal phase transforms into the cubic phase at $2370^{\circ} \mathrm{C}$. The cubic phase has moderate mechanical properties and is stable up to $2680^{\circ} \mathrm{C}$. The crystal phase transformation depends on temperature and is reversible. ${ }^{2}$ In order to maintain the tetragonal phase at room temperature after heating and fusion, stabilizing oxides $\left(\mathrm{CaO}, \mathrm{MgO}, \mathrm{CeO}_{2}, \mathrm{Y}_{2} \mathrm{O}_{3}\right)$ are added to zirconia crystals. ${ }^{20}$ However, because the tetragonal phase is only partially stabilized, ${ }^{21}$ unfavorable phase transformation from the tetragonal phase to the monoclinic phase may occur when external energy is provided. This change leads to crystal volume expansion of $3-4 \%$ at the involved area, which could be advantageous in resisting harmful crack propagation, but could cause unstable residual stress. ${ }^{22,23}$ Shape adjustments and finishing of zirconia in the clinic provides external energy to the zirconia crystal through local generation of heat and frictional force, which causes $m$ phase transformation. ${ }^{23}$

Although finishing and polishing procedures are important for clinical success, ${ }^{15,18}$ the effects of surface treatment protocols in combination with popular polishing systems have not been fully investigated. The purpose of this study was to evaluate the influence of surface treatment protocols and systems for finishing and polishing on the surface topography, bacterial adhesion, and phase transformation of monolithic zirconia. The first null hypothesis was that the surface treatment protocol would not have an influence on roughness of zirconia, regardless of the polishing systems used. The second null hypothesis was that use of different polishing systems on monolithic zirconia would not result in any significant differences in crystal structure or bacterial adhesion.

\section{MATERIALS AND METHODS}

Three hundred square-shaped $(20.0 \mathrm{~mm} \times 20.0 \mathrm{~mm} \times 2.8$ $\mathrm{mm})$ monolithic zirconia specimens were designed using computer software (CATIA V5R19, Dassault Systemes, Velizy-Villacoublay, France) and were manufactured with a 5-axis milling machine (Ceramill Motion 2, Amann Girrbach, Koblach, Austria) using zirconia blocks (Prettau, Zirkonzhan, $\mathrm{Au}$, Switzerland). The specimens were randomly assigned to three groups (100 specimens per group) according to the polishing system used: Jota (Jota kit 1434, Jota, Rüthi, Switzerland) (JO group), Meisinger (LUS85, Meisinger, Centennial, CO, USA) (ME group), and Edenta (Magic KIT Zir; Edenta GmbH, Lustenau, Austria) (ED group). The specimens for each system were further subdivided into four groups based on the surface treatment protocol used (25 specimens per protocol) (Fig. 1): coarse finishing alone (C protocol); coarse finishing and medium polishing (CM protocol); coarse finishing and fine polishing (CF protocol); coarse finishing, medium polishing and fine polishing (CMF protocol). All specimens were polished according to the protocols using an electronic low-speed handpiece ( $\mathrm{KaVO}$ EXPERTmatic E10 C, KaVo, Biberach, Germany) with backward movement at 20 seconds per step. The instruments and rpm used for the surface treatments are presented in Table 1.

\begin{tabular}{|c|c|c|c|}
\hline Protocol C & Coarse finishing & & \\
\hline Protocol CM & Coarse finishing & Medium polishing & \\
\hline Protocol CF & Coarse finishing & & Fine polishing \\
\hline Protocol CMF & Coarse finishing & Medium polishing & Fine polishing \\
\hline
\end{tabular}

Fig. 1. Surface treatment protocols.

Table 1. Finishing and polishing systems

\begin{tabular}{cccc}
\hline \multirow{4}{*}{ Group } & \multicolumn{3}{c}{ Instrument code (revolutions per minute) } \\
& Coarse finishing & Medium polishing & Fine polishing \\
& $(\mathrm{C})$ & $(\mathrm{M})$ & $(\mathrm{F})$ \\
\hline \multirow{2}{*}{ JO } & SZ652R.035.HP & ZIR9866M.040.HP & ZIR9866F.040.HP \\
& $(20.000)$ & $(8.500)$ & $(8.500)$ \\
ME & Z652R.104.HP & DCA04.104.HP & DCA10.104.HP \\
& $(10.000)$ & $(9.500)$ & $(9.500)$ \\
& 8001.050.HP & $3041 . \mathrm{HP}$ & $30041 . \mathrm{HP}$ \\
& $(12.500)$ & $(20.000)$ & $(10.000)$ \\
\hline
\end{tabular}

${ }^{\star}$ As provided by manufacturers

JO: Jota; ME: Meisinger; ED: Edenta 
The surface roughness $(\mathrm{Ra})$ values for zirconia specimens were determined using atomic force microscopy (AFM) (NX20, Park Systems, Suwon, Korea) and measurement software (Smartscan, Park Systems) using the noncontact mode at a scan rate of $1 \mathrm{~Hz}$ and $256 \times 250$ pixel resolution. The Ra for each specimen was calculated by averaging three measurements. The three-dimensional surface topography was visualized using the analysis software (XEI, Park Systems).

Structural studies of crystal phase transformation were performed for the three polishing systems using an X-ray diffractometer (XRD) (X'Pert PRO MPD, PANalytical, Almelo, The Netherlands). Diffraction patterns were obtained using $\mathrm{Cu}-\mathrm{Ka}$ radiation $(\lambda=1.5406 \AA)$ in the range of $20-40^{\circ}$ of $2 \theta$ with a step size of $0.016^{\circ}$ and step duration of $50.165 \mathrm{~s}$. The peaks were refined using pattern-decomposition and profile fitting functions of the software (HighScore Plus; PANalytical), which provided an asymmetric Pearson type VII function to distinguish among different phases. ${ }^{24}$ When the tetragonal $\mathrm{T}(101)$ peak at $2 \theta=29.807^{\circ}$ was asymmetric, the intensity of the peak resolved into individual peaks: $\mathrm{T}_{1}$ (refined $\mathrm{T}[101]$ ) and $\mathrm{T}_{2}$ (partially transformed T[101]) (Fig. 2). The monoclinic peak intensity ratio $\left(\mathrm{X}_{\mathrm{m}}\right)$ and the monoclinic volume $\left(\mathrm{V}_{\mathrm{m}}\right)$ were calculated as percentages with formulas (1) and (2) below: $:^{24,25}$

$$
\begin{gathered}
X_{m}=\frac{\mathrm{M}(-111)+\mathrm{M}(111)}{\mathrm{M}(-111)+\mathrm{M}(111)+\mathrm{T}_{1}} \\
V_{m}=\frac{1.311 \times \mathrm{X}_{\mathrm{m}}}{1+0.311 \times \mathrm{X}_{\mathrm{m}}}
\end{gathered}
$$

Where $\mathrm{M}(-111)$ and $\mathrm{M}(111)$ are the monoclinic peak intensities at $2 \theta=28.175^{\circ}$ and $31.468^{\circ}$, respectively; $T_{1}$ is the refined $\mathrm{T}(101)$ peak intensity.

The partially transformed tetragonal peak intensity ratio $\left(\mathrm{T}_{0}\right)$ was calculated with formula (3):

$$
T_{o}=\frac{\mathrm{T}_{2}}{\mathrm{~T}_{1}} \times 100 \%
$$

Streptococcus mutans (ATCC 25175) was used to assess biofilm formation. S. mutans was maintained on brain heart infusion (BHI) medium and grown under aerobic conditions. An in vitro biofilm formation assay was performed according to published protocol. ${ }^{26}$ Briefly, $S$. mutans colonies were inoculated into BHI-1\% sucrose broth and incubated overnight. The culture broth was inoculated into $3 \mathrm{~mL}$ of the same liquid medium to reach $1 \%(\mathrm{v} / \mathrm{v})$ in the 6 -well polystyrene plate containing each specimen. After incubation for 16 hours, streptococcal broth was removed, and the specimens were washed three times with sterile phosphatebuffered saline solution to remove loosely attached biomass. For biofilm quantification, $200 \mu \mathrm{L}$ of crystal violet solution $(0.2 \% \mathrm{w} / \mathrm{v}$ in $10 \%$ ethanol) was added to the specimens, followed by incubation for 1 hour. After washing with phosphate-buffered saline solution three times, the specimens were air-dried. Crystal violet retained by the streptococcal biofilm was redissolved in $200 \mu \mathrm{L}$ acidic solvent $(10 \%$ acetic acid in distilled water); absorbance was determined with a microplate reader (Molecular Devices; San Jose, CA, USA) at $595 \mathrm{~nm}$.

All statistical analyses were conducted using a statistical software package (IBM SPSS Statistics v22.0 for Windows; IBM Corp., Armonk, NY, USA). After determining normal distribution (Shapiro-Wilk test) and equal variances (Levene test), the results of surface roughness and bacterial adhesion were compared using one-way analysis of variance (ANOVA) and Tukey's test. Interactions between surface treatment protocols and polishing systems on the final roughness were statistically investigated with two-way ANOVA. Statistical significance was set at $P<.05$.

\section{RESULTS}

The surface roughness values of specimens treated by different surface treatment protocols and polishing systems are presented in Table 2 and Fig. 3. The CMF protocol resulted in significantly lower Ra values than did the other protocols

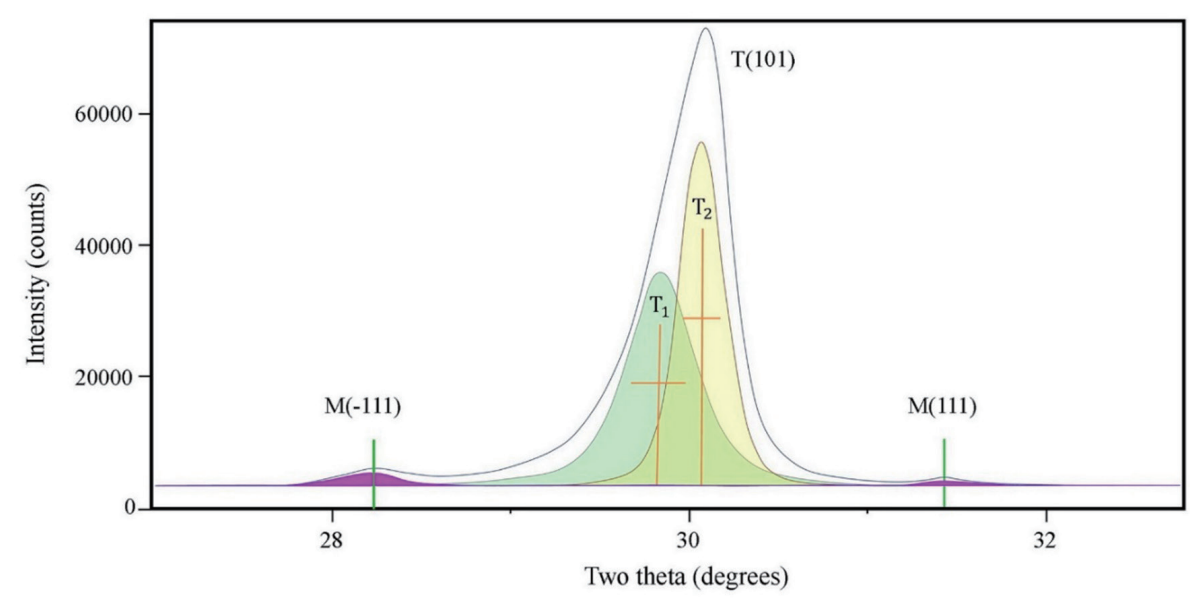

Fig. 2. Analyses of X-ray diffraction pattern of zirconia. $M(-111)$ : monoclinic peak at $28.175^{\circ} ; M(111)$ : monoclinic peak at $31.468^{\circ} ; \mathrm{T}(101)$ : tetragonal peak at $29.807^{\circ} ; \mathrm{T}_{1}$ : refined $\mathrm{T}(101) ; \mathrm{T}_{2}$ : partially transformed $\mathrm{T}(101)$. 
Table 2. Surface roughness ( $\mathrm{Ra}$ ) values of monolithic zirconia specimens after surface treatment

\begin{tabular}{cccccc}
\hline Group/subgroup & C & CM & CF & CMF & $P$ \\
\hline JO & $0.32(0.02)^{\mathrm{a}}$ & $0.16(0.07)^{\mathrm{b}}$ & $0.24(0.03)^{\mathrm{c}}$ & $0.05(0.07)^{\mathrm{d}}$ & $<.001$ \\
ME & $0.74(0.11)^{\mathrm{a}}$ & $0.09(0.08)^{\mathrm{b}}$ & $0.41(0.07)^{\mathrm{c}}$ & $0.08(0.03)^{\mathrm{b}}$ & $<.001$ \\
ED & $0.50(0.06)^{\mathrm{a}}$ & $0.29(0.03)^{\mathrm{b}}$ & $0.44(0.07)^{\mathrm{a}}$ & $0.09(0.04)^{\mathrm{c}}$ & $<.001$ \\
\hline
\end{tabular}

* Data are presented as mean (standard deviation) unless otherwise indicated.

+ Same superscript lowercase letters indicate there is no statistically significant difference within row.

JO: Jota; ME: Meisinger; ED: Edenta; C: coarse finishing alone; CM: coarse finishing and medium polishing; CF: coarse finishing and fine polishing; CMF: coarse finishing, medium polishing, and fine polishing.
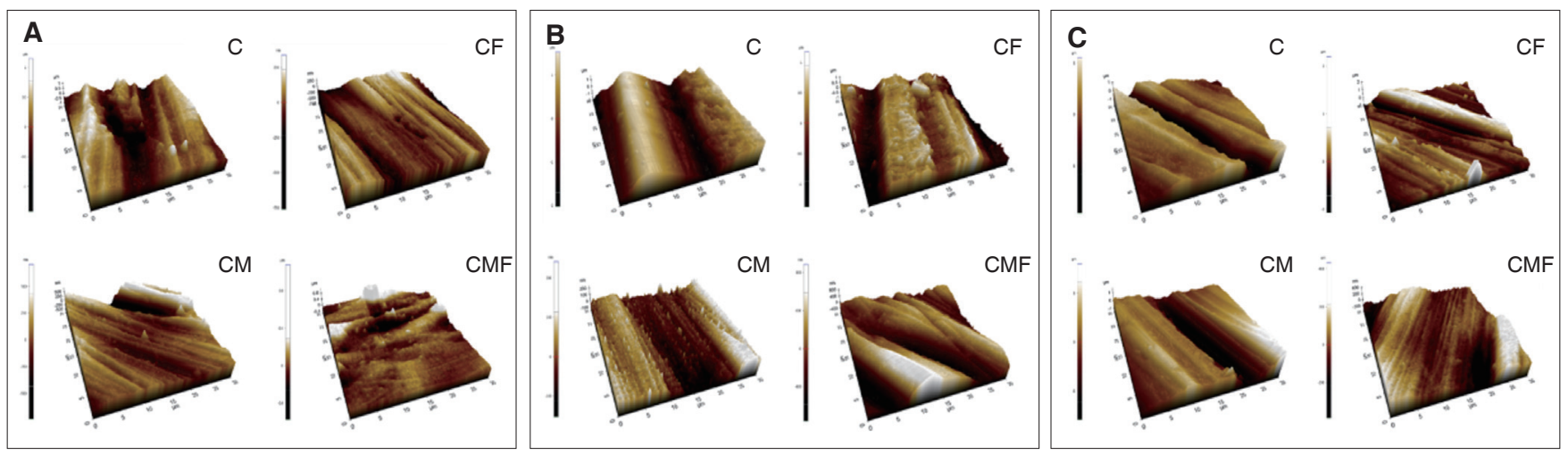

Fig. 3. Three-dimensional topography of monolithic zirconia specimens after surface treatment. (A) Jota; (B) Meisinger; (C) Edenta. C: coarse finishing alone; CM: coarse finishing and medium polishing; CF: coarse finishing and fine polishing; CMF: coarse finishing, medium polishing, and fine polishing.

Table 3. Variations between surface treatment protocols and polishing systems, results of two-way analysis of variance

\begin{tabular}{lccccc}
\hline \multicolumn{1}{c}{ Source } & Type III sum of squares & Degrees of freedom & Mean Square & $F$ & $P$ value \\
\hline Polishing system & .051 & 2 & .025 & .118 & .785 \\
Polishing protocol & .354 & 3 & .014 & 8.254 \\
System $\times$ protocol & .086 & 6 & & .015 \\
Error & .000 & 0 & & \\
Total & 1.452 & 12 & & \\
Correct total & .490 & 11 & & \\
\hline
\end{tabular}

for $\mathrm{JO}$ and ED groups $(P<.001)$. ME group also showed lower Ra values in CMF protocol than in CM protocol, but the difference was not statistically different. The CM protocol resulted in lower $\mathrm{Ra}$ values than did the $\mathrm{CF}$ protocol $(P$ $<$.001). In comparing finishing and polishing systems, the JO group generally exhibited the lowest roughness values, followed by the ME and ED groups. Two-way ANOVA showed significant interaction between the surface treatment protocol and the polishing system on the resulting roughness $(P<.001)$ (Table 3$)$.

The XRD patterns for each system after use of the $\mathrm{CMF}$ protocol are presented in Figure 4. The major peaks of the tetragonal phase were located at $30.1^{\circ}(2 \theta)$, corresponding to the orientation $\mathrm{T}(101)$. For the monoclinic phase, two peaks were observed at $28.2^{\circ}$ and $31.2^{\circ}(2 \theta)$, corresponding to orientation $\mathrm{M}(111)$ and $\mathrm{M}(-111) . \mathrm{X}_{\mathrm{m}}, \mathrm{V}_{\mathrm{m}}$, and $\mathrm{T}_{0}$ values for specimens are presented in Table 4 . The JO group exhibited the smallest $\mathrm{X}_{\mathrm{m}}$ and $\mathrm{V}_{\mathrm{m}}$, followed by the $\mathrm{ED}$ and $\mathrm{ME}$. The $\mathrm{T}_{0}$ ranged from $60.58 \%$ to $85.96 \%$. The smallest $\mathrm{T}_{0}$ was found on the specimens in the ME group, while the highest ratio was found on specimens in the ED group.

In vitro streptococcal biofilm formation on specimen surfaces after use of the CMF protocol were compared among systems (streptococcal biofilm with crystal violet staining) (Fig. 5). S. mutans biofilm formation increased significantly in ED group specimens, followed by $\mathrm{ME}$ and JO group ( $P$ $<$.001) (Fig. 6). 


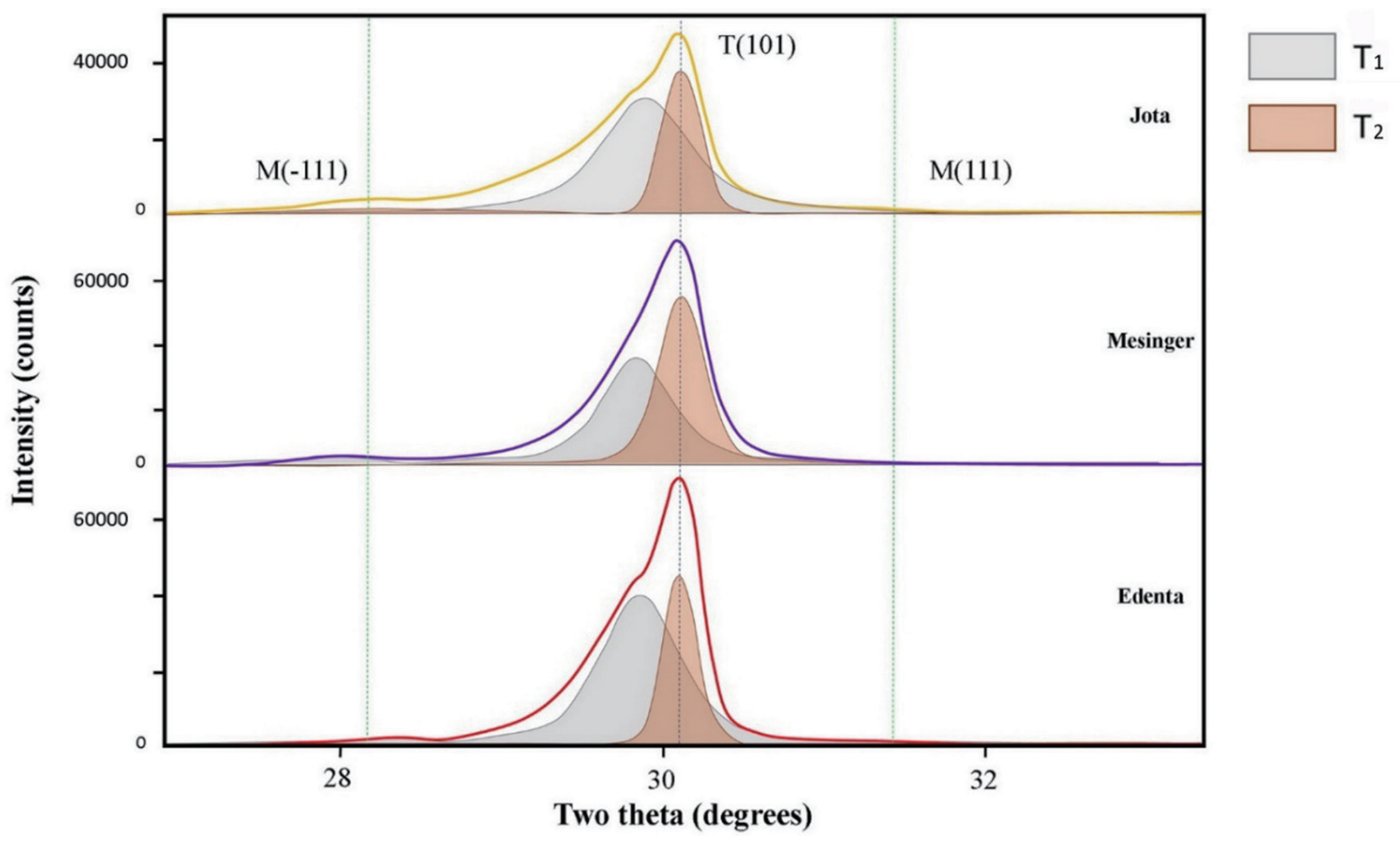

Fig. 4. X-ray diffraction patterns after coarse finishing, medium polishing, and fine polishing, by polishing system. $\mathrm{M}(-111)$ : monoclinic peak at $28.175^{\circ} ; \mathrm{M}(111)$ : monoclinic peak at $31.468^{\circ} ; \mathrm{T}(101)$ : tetragonal peak at $29.807^{\circ} ; \mathrm{T}_{1}$ : refined $\mathrm{T}(101) ; \mathrm{T}_{2}$ : partially transformed $\mathrm{T}(101)$.

Table 4. The monoclinic peak intensity ratio $\left(\mathrm{X}_{\mathrm{m}}\right)$, monoclinic volume $\left(\mathrm{V}_{\mathrm{m}}\right)$, and partially transformed tetragonal peak intensity ratio $\left(\mathrm{T}_{0}\right)$ of specimens, by polishing system

\begin{tabular}{cccc}
\hline Finishing and polishing system & \multicolumn{3}{c}{ Relative amount (\%) } \\
& $X_{m}$ & $V_{m}$ & $T_{0}$ \\
\hline JO & 3.68 & 2.25 & 68.99 \\
ME & 4.38 & 2.43 & 60.58 \\
ED & 3.71 & 2.26 & 85.96 \\
\hline
\end{tabular}

JO: Jota; ME: Meisinger; ED, Edenta.
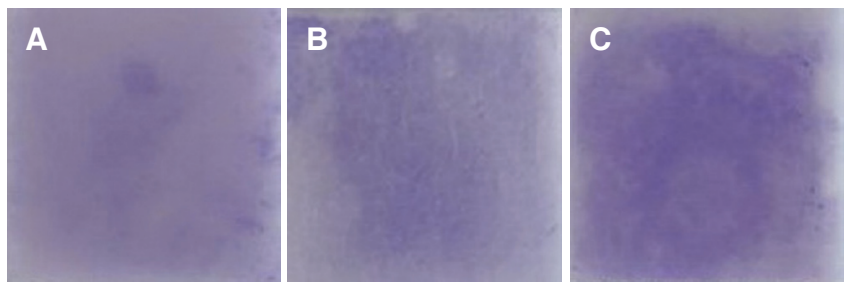

Fig. 5. Crystal violet staining of streptococcal biofilm, by polishing system. (A) Jota, (B) Meisinger, (C) Edenta.

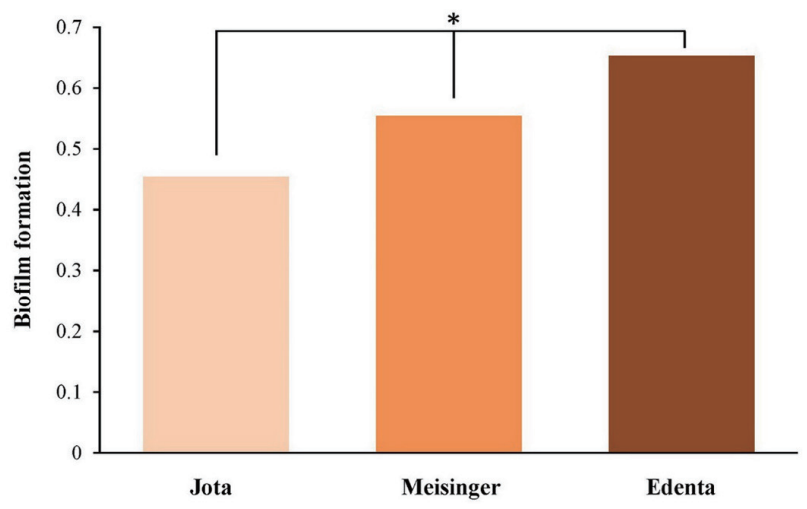

Fig. 6. Biofilm formation determined by crystal violet staining, by polishing system. *Statistical significance. 


\section{DISCUSSION}

This study investigated the surface topography, crystal structure change, and biofilm formation of monolithic zirconia treated by various finishing and polishing protocols and systems. Our results showed that differences in instrumentation protocols and systems led to different roughness, crystal phase transformation, and bacterial adhesion characteristics. Thus, the first and second null hypotheses were rejected.

Determining a proper surface treatment protocol to produce smooth surfaces with a limited time, instruments, and application steps has become a crucial point of interest. ${ }^{27-29}$ Preis et al. conducted research on 14 different commercial zirconia polishing kits and found that the unequal effectiveness of different polishing systems may not be affected by the number of polishing steps but may depend on the sequence of those steps. ${ }^{15}$ In this study, omission of a polishing step significantly influenced the surface roughness. Clearly, a complete polishing protocol without omission of any of the polishing steps produced the best surface smoothness for zirconia. Coarse-grit finishing and medium-grit polishing burs were essential for smoothing deep grooves and scratches. When the medium-grit polishing bur was omitted, use of the fine-grit polishing bur alone did not effectively reduce roughness. Sequential application of polishing steps, with focus on coarse-grit finishing and medium-grit polishing burs, was a key to achieving a smooth surface.

The overlapping peaks observed on XRD demonstrate an intrinsic problem in analyzing the crystal structure of a material. Zirconia exhibits a phase transformation between monoclinic, tetragonal, and cubic phases. Taking into account coexistence between monoclinic and tetragonal phases in the XRD pattern of zirconia after surface treatment, elucidating contributions of each of these different phases is essential to accurate analysis of a complex XRD profile. ${ }^{30}$ The phase transformation of zirconia after surface treatment has been evaluated in numerous studies. ${ }^{13,18,19,31} \mathrm{In}$ general, the $\mathrm{m} \rightarrow \mathrm{t}$ peak was endothermically symmetrical, but the $\mathrm{t} \rightarrow \mathrm{m}$ peak was asymmetrical with an abrupt side. ${ }^{30}$ Based on results of classical and simple XRD analysis methods, the phase quantification underestimated the monoclinic phase by overestimating the tetragonal proportion. Through use of profile fitting and decomposition methods, all peaks are properly included, and the contributions of the overlapping peaks were accurately separated. Therefore, this study described not only the transformation trend, but also provided more precise peak analyses regarding the position, intensity and shape of each individual peak in diffraction patterns.

Oral bacterial biofilm formation on the surfaces of restorations lead to surface biodegradation, secondary caries, gingivitis, and mouth odor. ${ }^{32}$ Surface roughness has been identified as the most important factor in biofilm formation. ${ }^{9}$ Based on the results of this study, the rougher surfaces exhibited higher initial bacteria formation. Correlation of surface roughness and bacterial adhesion could be explained by the theory of bacterial adhesion and retention. ${ }^{33}$ There are four phases of bacterial adhesion, including transportation of the bacterium toward the surface, initial bacterial adhesion, attachment by specific interactions, and colonization of the surface. ${ }^{33}$ Physically, the initial bacterial adhesion requires interaction between bacteria and the surface from a distance of approximately $50 \mathrm{~nm}$ through a combination of electrostatic attractive and repulsive forces. Rough surfaces exhibit irregular geometry in which bacteria are strongly protected against shear forces and provide favorable circumstances for bridging distances. ${ }^{9}$ Thus, attachment is more strongly established on a rough surface.

The final characteristic of the zirconia surface is influenced by extrinsic and intrinsic factors. This article included surface treatment protocols and commercial finishing and polishing systems as experimental factors, which are clinically important extrinsic interventions. The grinding modes and types of the rotary handpiece are other extrinsic factors that affect the results of finishing and polishing. The zirconia block itself is a fundamental intrinsic factor that should be considered when evaluating a surface finishing and polishing method. ${ }^{34}$ Thus, these factors should be included in the study design of future investigations. Moreover, largesize clinical prospective trials will be needed to confirm the results of the current in vitro study.

\section{CONCLUSION}

This study demonstrated that differences in surface treatment protocols and polishing systems led to different roughness, crystal phase transformation, and bacterial adhesion characteristics. Sequential application of polishing steps, focusing on use of both coarse-grit finishing and medium-grit polishing burs, was a key to achieving a smooth surface. Moreover, special consideration should be given to selecting a commercial polishing system.

\section{ORCID}

Hang-Nga Mai https://orcid.org/0000-0002-9832-3312

Su-Hyung Hong https://orcid.org/0000-0001-5569-3826

Sung-Hun Kim bttps://orcid.org/0000-0003-3289-9703

Du-Hyeong Lee https://orcid.org/0000-0003-2803-7457

\section{REFERENCES}

1. Miyazaki T, Nakamura T, Matsumura H, Ban S, Kobayashi T. Current status of zirconia restoration. J Prosthodont Res 2013;57:236-61.

2. Kelly JR, Denry I. Stabilized zirconia as a structural ceramic: an overview. Dent Mater 2008;24:289-98.

3. Guazzato M, Albakry M, Ringer SP, Swain MV. Strength, fracture toughness and microstructure of a selection of allceramic materials. Part II. Zirconia-based dental ceramics. Dent Mater 2004;20:449-56.

4. Hmaidouch R, Müller WD, Lauer HC, Weigl P. Surface roughness of zirconia for full-contour crowns after clinically simulated grinding and polishing. Int J Oral Sci 2014;6:241-6. 
5. Khayat W, Chebib N, Finkelman M, Khayat S, Ali A. Effect of grinding and polishing on roughness and strength of zirconia. J Prosthet Dent 2018;119:626-31.

6. Alao AR, Stoll R, Song XF, Miyazaki T, Hotta Y, Shibata Y, Yin L. Surface quality of yttria-stabilized tetragonal zirconia polycrystal in CAD/CAM milling, sintering, polishing and sandblasting processes. J Mech Behav Biomed Mater 2017;65: 102-16.

7. Kim MJ, Oh SH, Kim JH, Ju SW, Seo DG, Jun SH, Ahn JS, Ryu JJ. Wear evaluation of the human enamel opposing different Y-TZP dental ceramics and other porcelains. J Dent 2012;40:979-88.

8. Teughels W, Van Assche N, Sliepen I, Quirynen M. Effect of material characteristics and/or surface topography on biofilm development. Clin Oral Implants Res 2006;17:68-81.

9. Sagsoz O, Demirci T, Demirci G, Sagsoz NP, Yildiz M. The effects of different polishing techniques on the staining resistance of CAD/CAM resin-ceramics. J Adv Prosthodont 2016;8:417-22.

10. Amer R, Kürklü D, Johnston W. Effect of simulated mastication on the surface roughness of three ceramic systems. J Prosthet Dent 2015;114:260-5.

11. Etman MK, Woolford M, Dunne S. Quantitative measurement of tooth and ceramic wear: in vivo study. Int J Prosthodont 2008;21:245-52.

12. Mohammadi-Bassir M, Babasafari M, Rezvani MB, Jamshidian M. Effect of coarse grinding, overglazing, and 2 polishing systems on the flexural strength, surface roughness, and phase transformation of yttrium-stabilized tetragonal zirconia. J Prosthet Dent 2017;118:658-65.

13. Jones CS, Billington RW, Pearson GJ. The in vivo perception of roughness of restorations. Br Dent J 2004;196:42-5.

14. Preis V, Grumser K, Schneider-Feyrer S, Behr M, Rosentritt M. The effectiveness of polishing kits: influence on surface roughness of zirconia. Int J Prosthodont 2015;28:149-51.

15. Caglar I, Ates SM, Yesil Duymus Z. The effect of various polishing systems on surface roughness and phase transformation of monolithic zirconia. J Adv Prosthodont 2018;10: $132-7$.

16. Jefferies SR. Abrasive finishing and polishing in restorative dentistry: a state-of-the-art review. Dent Clin North Am 2007;51:379-97, ix.

17. Al-Haj Husain N, Özcan M. A study on topographical properties and surface wettability of monolithic zirconia after use of diverse polishing instruments with different surface coatings. Prosthodont 2018;27:429-42.

18. Huh YH, Park CJ, Cho LR. Evaluation of various polishing systems and the phase transformation of monolithic zirconia. J Prosthet Dent 2016;116:440-9.

19. Kobayashi K, Kuwajima H, Masaki T. Phase change and mechanical properties of $\mathrm{ZrO}_{2}-\mathrm{Y}_{2} \mathrm{O}_{3}$ solid electrolyte after ageing. Solid State Ion 1980;3-4:489-93.

20. Gibson IR, Irvine JT. Qualitative X-ray diffraction analysis of metastable tetragonal $\left(\mathrm{t}^{\prime}\right)$ zirconia. J Am Ceram Soc 2001; 84:615-8.

21. Albakry M, Guazzato M, Swain MV. Effect of sandblasting, grinding, polishing and glazing on the flexural strength of two pressable all-ceramic dental materials. J Dent 2004;32:919.

22. Luthardt RG, Holzhüter M, Sandkuhl O, Herold V, Schnapp JD, Kuhlisch E, Walter M. Reliability and properties of ground Y-TZP-zirconia ceramics. J Dent Res 2002;81:487-91.

23. Toraya H, Yoshimura M, Somiya S. Calibration curve for quantitative analysis of the monoclinic-tetragonal $\mathrm{ZrO}_{2}$ system by X-ray diffraction. J Am Ceram Soc 1984;67:C119-21.

24. Garvie RC, Nicholson PS. Phase analysis in zirconia systems. J Am Ceram Soc 1972;55:303-5.

25. Gilan I, Sivan A. Extracellular DNA plays an important structural role in the biofilm of the plastic degrading actinomycete Rhodo-coccus ruber. Adv Microbiol 2013;3:543-51.

26. Silva TM, Salvia AC, Carvalho RF, Pagani C, Rocha DM, Silva EG. Polishing for glass ceramics: which protocol? J Prosthodont Res 2014;58:160-70.

27. Jing Z, Ke Z, Yihong L, Zhijian S. Effect of multistep processing technique on the formation of micro-defects and residual stresses in zirconia dental restorations. J Prosthodont 2014;23:206-12.

28. Erdemir U, Sancakli HS, Yildiz E. The effect of one-step and multi-step polishing systems on the surface roughness and microhardness of novel resin composites. Eur J Dent 2012;6: 198-205.

29. Lanson B, Velde B. Decomposition of X-ray diffraction patterns: a convenient way to describe complex I/S diagenetic evolution. Clays Clay Miner 1992;40:629-43.

30. Park C, Vang MS, Park SW, Lim HP. Effect of various polishing systems on the surface roughness and phase transformation of zirconia and the durability of the polishing systems. J Prosthet Dent 2017;117:430-7.

31. Lee BC, Jung GY, Kim DJ, Han JS. Initial bacterial adhesion on resin, titanium and zirconia in vitro. J Adv Prosthodont 2011;3:81-4.

32. Bollen CM, Lambrechts P, Quirynen M. Comparison of surface roughness of oral hard materials to the threshold surface roughness for bacterial plaque retention: a review of the literature. Dent Mater 1997;13:258-69.

33. Kim HK, Kim SH. Comparison of the optical properties of pre-colored dental monolithic zirconia ceramics sintered in a conventional furnace versus a microwave oven. J Adv Prosthodont 2017;9:394-401.

34. Choi JW, Kim SY, Bae JH, Bae EB, Huh JB. In vitro study of the fracture resistance of monolithic lithium disilicate, monolithic zirconia, and lithium disilicate pressed on zirconia for three-unit fixed dental prostheses. J Adv Prosthodont 2017;9: 244-51. 